\title{
MOTION COMPENSATION FOR HAND HELD CAMERA DEVICES
}

\author{
Dhara Patel $^{1}$, Dixesh Patel ${ }^{2}$, Devang Bhatt ${ }^{3}$, Kalpesh R Jadav ${ }^{4}$ \\ ${ }^{1}$ P.G.Student, Parul Institute of Engineering \& Technology, Gujarat, India \\ ${ }^{2}$ P.G.Student Parul Institute of Engineering \& Technology, Gujarat, India \\ ${ }^{3}$ P.G.Student, Parul Institute of Engineering \& Technology, Gujarat, India \\ ${ }^{4}$ Department of electronics and communication engineering, Parul institute of engineering \& technology, Vadodara, \\ India
}

\begin{abstract}
With handy camera image is not enough stable at that time stabilization method is used to recover that shaky effect. So, stabilization of image is concept to recover the scale and theta of shaky image. For that algorithm should be able to stabilize the image with maximum original information from that shaky input image. And from this image stabilization algorithm we can use this as a fundamental concept to stabilize the video. Here in this paper algorithm is applied for $2 D$ image and measure the efficiency of that algorithm
\end{abstract}

Keywords: Motion estimation; Feature detection methods; FAST feature detection

\section{INTRODUCTION}

Stabilization concept is used to stabilize the image for further use for detecting object and its post processing work. With hand-held cameras the output is not stabilize with respect to ground truth. For image stabilization we having three type of stabilization environment methods [1] given as, 1. Optical image stabilization, 2.Mechanical stabilization and 3. Digital image stabilization. Here in this paper digital image stabilization is used. In method of optical image stabilization is held by shifting the IS lens group on a plane perpendicular to the optical axis to counter the degree of image vibration, the light rays reaching the image plane can be steadied [2]. Mechanical image stabilization involves stabilizing the entire camera, not just the image. This type of stabilization uses a device called "Gyros". Gyros consist of a gyroscope with two perpendicular spinning wheels and a battery pack [3]. Gyroscopes are motion sensors. When the gyroscopes sense movement, a signal is sent to the motors to move the wheels to maintain stability. The gyro attaches to the camera's tripod socket and acts like an "invisible tripod" [3]. In digital image stabilization method that involves mainly three steps for 2D image stabilization [4] which are involved motion estimation, motion compensation and image compression for the stabilized output. This digital image stabilization is a post processing technique. It is the process of generating a compensated video sequence where any and all unwanted camera motion is subtracted from the original input. Most proposed DIS systems compensate for all motion [ 4,5,8], producing a sequence where the background remains motionless. Other techniques only subtract the $3 \mathrm{D}$ rotation of the camera [6,7], generating a denoted sequence.

The paper is organised as follows: in section II. Related work of image stabilization is given from reference papers, in section III. Discussed concept estimation of motion, and compensation concept, and in section IV. About algorithm of proposed work is given and in last section we discuss about conclusion from regarding results from related algorithm.

Here the general model of image stabilization is given: Basic model [4] of image stabilization for the system described [7]. That consist of three stages 1. Motion estimation, in that motion points and static points are considered into account. That is the main concept of feature extraction from reference image, in stage 2. Motion compensation which rejects the outliers points and third is to compress the image.

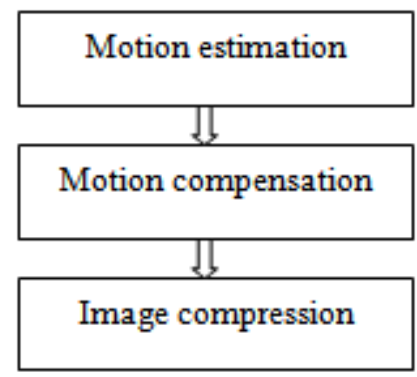

Fig-1: Block diagram of image stabilization [4]

The three given parameter Euclidean model compensates for translations and rotation around the optical axis. The similarity model has an extra parameter to include scaling; and the affine transform model requires 6 parameters to accommodate different horizontal and vertical scaling, and skewing. [4]

\section{RELATED WORK:}

The goal of feature based algorithms is to estimate inter frame motion by extraction features from video images [9]. Some techniques [10] that combine features extraction with other robust filters have good performances. Motion 
filtering is also a significant step in video stabilization process. In this step undesired movement is recognized by evaluation of estimated motion. Different methods have been introduced to correct translational and rotational jitters. Kalman filtering [11] and extended Kalman filtering, Frame Position Smoothing [13], Gaussian filtering [15] and Motion Vector Integration [14] are among these techniques. A video stabilization algorithm using SIFT [12] has been introduced in [14]. Julian et al [14] uses Iterative Least Squares method to reduce estimation error then uses Adaptive Motion Vector Integration to filter intentional camera motion. Another system [15] employs SIFT point in order to calculate inter frame motion. They recognize intentional movement by Kalman filtering and reduce error variance by using particle filter. But, in this system original SIFT algorithm's parameters doesn't adapt to video stabilization system. And both Kalman filtering and particle filtering calculated for each frame implies intensive computation.

\section{MOTION ESTIMATION:}

To separate the desire and undesired motion algorithm should be based on motion estimation. So for any video stabilization the motion estimation is the basic step towards the stabilization technique. Motion estimation having two types of interested points which are static and dynamic. So from reference of static we can make estimation of dynamic points. And that is the core idea of any stabilization method. In motion estimation technique feature based approach or direct pixel base approach. Here in this paper, algorithm is for feature based approach technique.

In this paper the motion between two sequential frames are, $\mathrm{f}(\mathrm{x}, \mathrm{y}, \mathrm{t})$ and $\mathrm{f}(\mathrm{x}, \mathrm{y}, \mathrm{t}-1)$ is model with a 6-parameter affine transform. The motion vectors between two images can be described by a single affine transformation as given below by [1] eq. (1). Where $\mathrm{m} 1, \mathrm{~m} 2, \mathrm{~m} 3, \mathrm{~m} 4$ represents the $2 \times 2$ affine rotation matrix $\mathrm{A}$, and $\mathrm{m} 5$ and $\mathrm{m} 6$ the translation vector $\bar{T}$.

$f\left(x_{v}, t\right)=f\left(a_{1} x+a_{2} y+, a_{a} x+a_{4} y+a_{6}, t-1\right)$ Eq (1)

Where

$A=\left(\begin{array}{ll}a_{1} & a_{2} \\ a_{a} & a_{4}\end{array}\right)$ And $\bar{T}=\left(\begin{array}{l}a_{5} \\ a_{6}\end{array}\right)$

In the order to estimate the affine parameters, we define the following quadratic errors function [1] to be minimized.

$E(a)=\Sigma_{t}\left[f\left(x_{v} y, t\right)-f\left(a_{1} x+a_{2} y+a_{5}, a_{a} x+a_{4} y+\right.\right.$ $\left.\left.a_{6,} t-1\right)\right]^{2}$

Where symbol $\Omega$ denotes a user specified region of interest here it is the entire frame. Since this error function is nonlinear and its affine parameters $\mathrm{m}$, cannot be minimized analytically. To simplify the minimization, this error function is approximated by using a first-order truncated Taylor series expansion. The quadratic error function is now in linear form, its unknowns, $m$ and can therefore be minimized analytically by differentiating with respect to $m$ as shown in eq.9[1].

$$
\frac{d E(a)}{d a}=\Sigma_{\Omega} 2 C\left[k-C^{T} a\right]
$$

The temporal derivatives can be derived using Separable filters as mentioned in [1]. Finally L-level

\section{FEATURE DETECTION METHODS:}

BRIFF (Binary Robust Independent Elementary Features): this technique [17] as an efficient descriptor for key points. This descriptor is built by simple binary tests on a subset of the pixels surrounding the key point centre.

RIFF: (Rotation-Invariant, Fast Feature): introduced by [19], based on a HOG computed at a circular support area and used an annular binning to achieve orientation invariance.

SURF: (Speeded up Robust Features): introduced by [16] and based on Fast-Hessian Detector to find Key points. This descriptor mainly focuses on reducing computational time.

ORB: (Oriented FAST and Rotated BRIEF): introduced by [18] ORB detects key points by adding a fast and accurate orientation component, and uses the rotated BRIEF descriptor.

\section{FAST FEATURE DETECTION TECHNIQUE:}

Feature detection technique [20] is given in below graph. Here the comparisons are based on detection cost [20] and number of detection features [20]. In both the cases FAST feature technique is better for slow motion camera inputs.

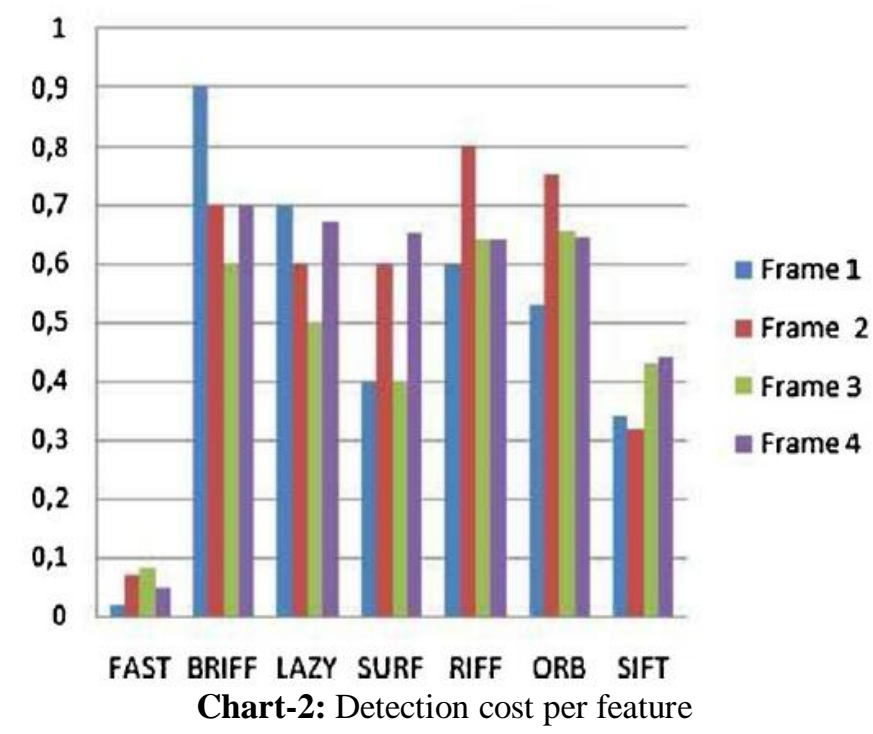




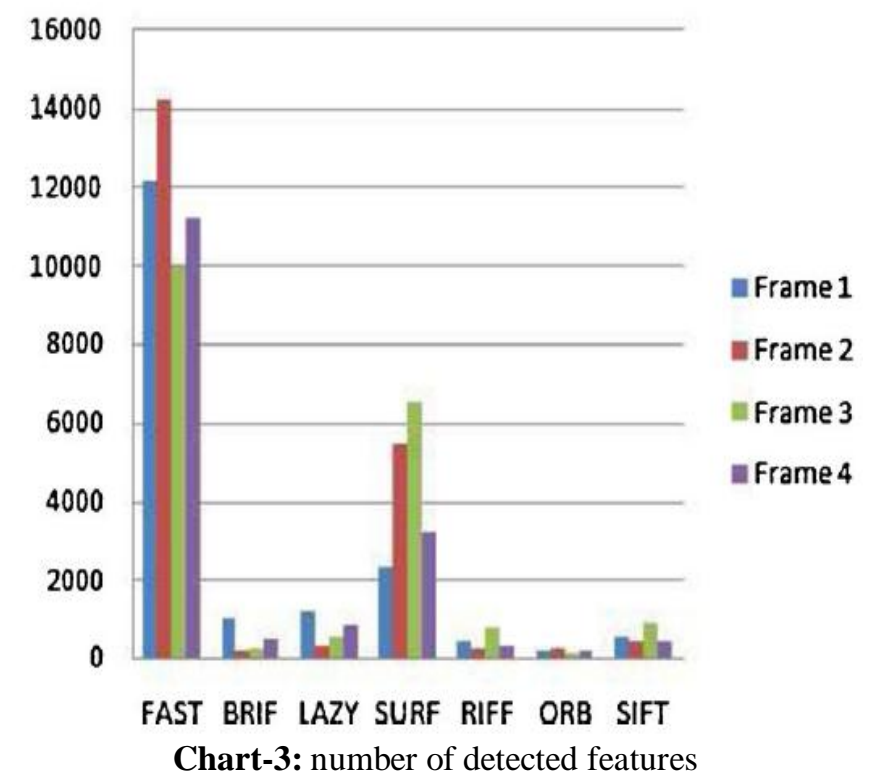

Regarding computational efficiency, in Fig. 2, SURF requires approximately $0.4 \mathrm{~ms}$ to detect a feature. The runtime of FAST technique is under $0.1 \mathrm{~ms}$ per feature while that of SIFT technique comes to $0.5 \mathrm{~ms}$ per feature and other methods compute each feature in around $0.6 \mathrm{~ms}$.[20]. So we can conclude that FAST feature technique is better than any other technique for handheld camera devices. So we used fast feature technique in our proposed method.

\section{PROPOSED ALGORITHM FOR IMAGE STABILIZATION:}

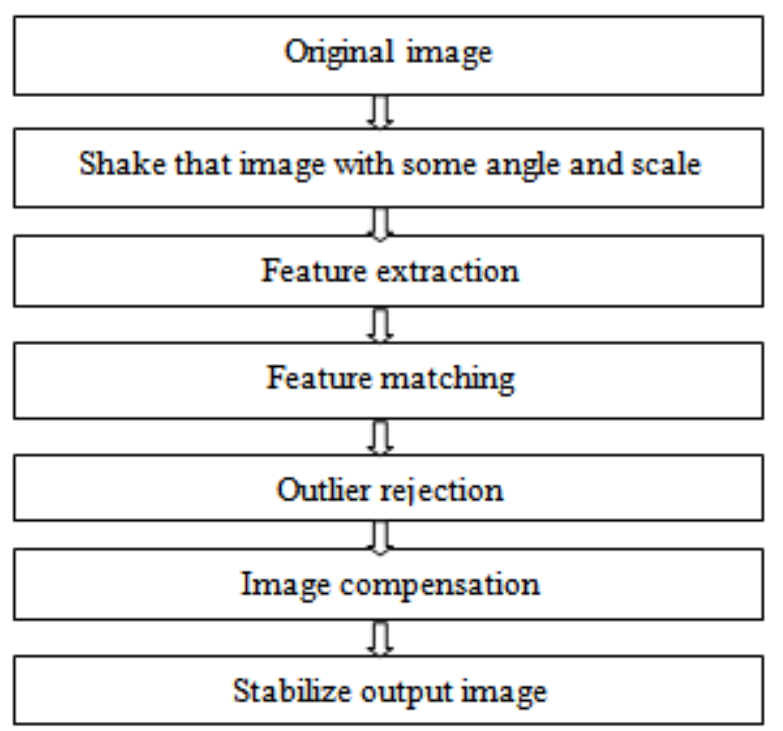

Fig-2: proposed algorithm

\section{RESULTS}
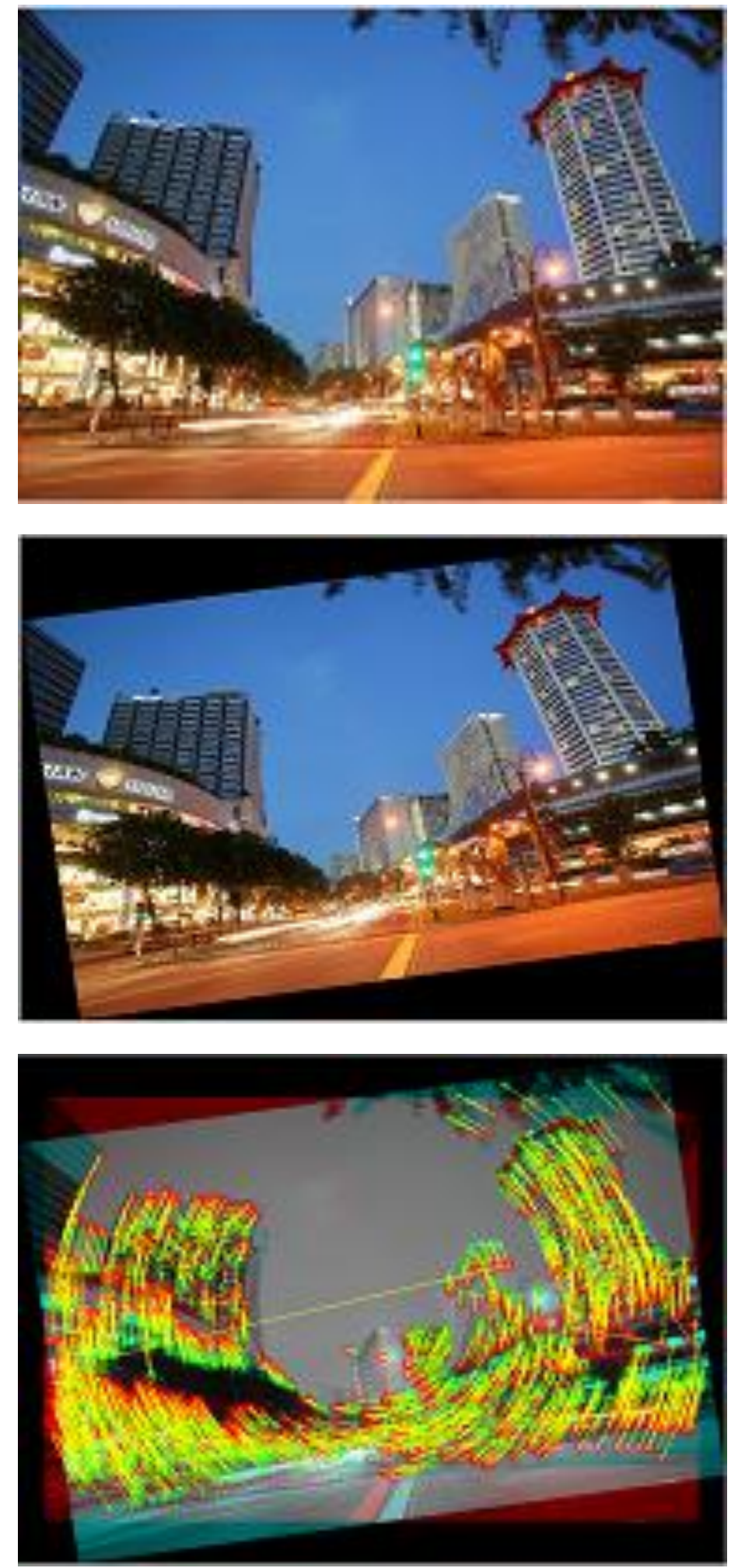

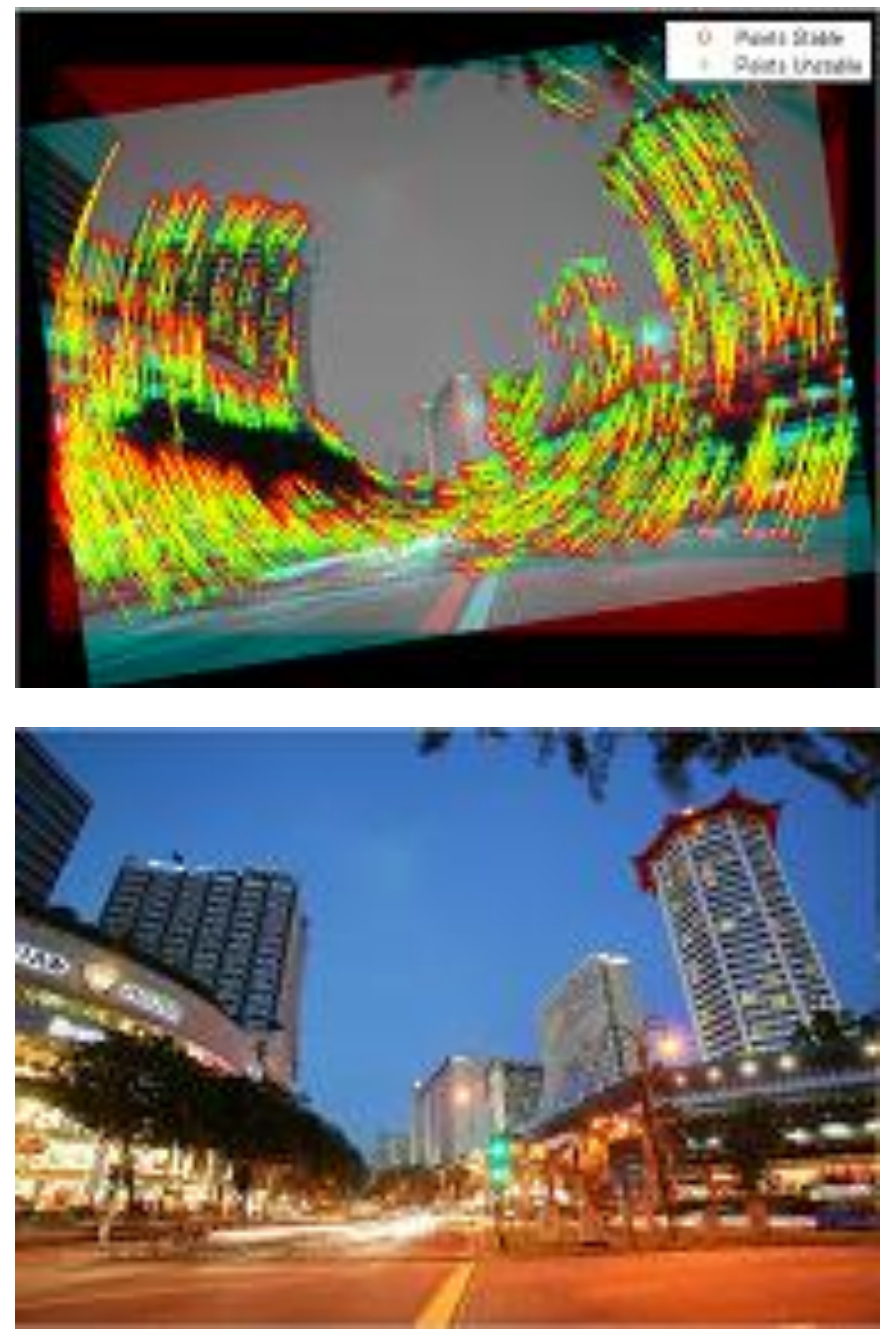

\section{CONCLUSION AND FUTURE WORK:}

In this paper, we proposed a method for compensation of motion with feature based technique. This technique is work for stabilization of extracting features based on FAST feature extraction and matching technique. That is faster compare to other methods and collect more features also. We demonstrate that FAST feature technique is more robust for hand held camera devices. For future work we focused on following points

- To increase more accuracy for collecting the features extraction and matching.

- To increase robustness for high quality images.

- To remove the noise from the images and then stabilize it.

- Applied this method for video stabilization on hand held camera devices.

\section{REFERENCES}

[1]. Paresh Rawat, Jyoti Singhai "Review of Motion Estimation and Video Stabilization techniques For hand held mobile video" Signal \& Image Processing : An International Journal (SIPIJ) Vol.2, No.2, June 2011 DO: $10.5121 /$ sipij.2011.2213 159
[2]. Canon Digisuper 100xs, Product Manual, [online] URL http://www.canon.com/bctvproducts/pdf/1 00xs.pdf [Accesses 20 May 2009]

[3]. Multimedia, "Use Image Stabilize. For Gyroscopic Stabilizer", [online],

URLhttp://www.websiteoptimization.com/speed/tweak/stabi lizer. Access 13-January- 2009].

[4]. Carlos Morimoto and Rama Chellappa, "Evaluation of image stabilization algorithms," in Acoustics, Speech and Signal Processing, 1998. Proceedings of the 1998 IEEE International Conference on, 1998, vol. 5, pp. 2789-2792.

[5]. M. Irani, B. Rousso, and S. Peleg." Recovery of egomotion using image stabilization". In Proc. IEEE Conference on Computer Vision and Pattern Recognition, pages 454-460, Seattle, WA, June 1994.

[6] Z.Duri'c and A. Rosenfeld. "Stabilization of image sequences." Technical Report CAR-TR-778, Centre for Automation Research, University of Maryland, College Park, 1995.

[7]. C.H. Morimoto and R. Chellappa. "Fast 3d stabilization and mosaic king." In Proc. IEEE Conference on Computer Vision and Pattern Recognition, Puerto Rico, PR, June 1997.

[8]. C.H. Morimoto and R. Chellappa. "Fast electronic digital image stabilization". In Proc. International Conference on Pattern Recognition, Vienna, Austria, August 1996.

[9]. Wali A and Alimi AM, "Incremental learning approach for events detection from large video dataset," Advanced Video and Signal Based Surveillance (AVSS), pages 555 560,2010

[10]. Lu W, Hongying Z, Shiyi G, Ying M and Sijie L, "The adaptive compensation algorithm for small UAV image stabilization", Geosciences and Remote Sensing Symposium (IGARSS), pages 4391-4394, 2012.

[11]. Erturk S. "Image sequence stabilisation based on kalman filtering of frame positions". Electronics Letters, 37(20), 2008.

[12]. Lowe D. Distinctive " image features from scaleinvariant keypoints". International Journal of Computer Vision, Vol. 60(2), pages 91-110, 2004.

[13]. Erturk S," Image sequence stabilisation: motion vector integration (MVI) versus frame position smoothing (FPS) Image and Signal Processing and Analysis", pages 266-271, 2001.

[14]. Junlan Y, Schonfeld D and Mohamed M, "Robust Video Stabilization Based on Particle Filter Tracking of Projected Camera Motion, Circuits and Systems for Video Technology", IEEE Transactions on , vol.19, no.7, pages 945-954, 2009.

[15]. Hong S and Atkins E, "Moving Sensor Video Image Processing Enhanced with Elimination of Ego Motion by Global Registration and SIFT, Tools with Artificial Intelligence", ICTAI '08, poges37-40, 2008.

[16]. Bay H, Tuytelaars T, Gool LV (2006) "Surf: speeded up robust features". In: ECCV. pp 404-417

[17]. CalonderM, Lepetit V, Strecha C, Fua P (2010) "Brief: binary robust independent elementary features". In:Computer vision ECCV 2010. Lecture notes in computer science, vol 6314. Springer, Berlin Heidelberg, pp 778-792 
[18]. Rublee E, Rabaud V, Konolige K, Bradski G (2011) Orb: an efficient alternative to siftor surf. In: 2011 IEEE international conference on computer vision (ICCV). pp 2564 2571.doi:10.1109/ICCV.2011.6126544

[19]. Rotation invariant fast features for large-scale recognition, vol 8499 (2012). doi:10.1117/12.945968

[20]. Ahlem Walha, Ali Wali, Adel M. Alimi "Video stabilization with moving object detecting and tracking for aerial video surveillance" Springer Science Business Media New York 2014

\section{BIOGRAPHIES}

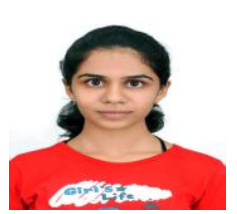

I have completed my B.E. of Electronics \& Communication Engineering in Gujarat Technological University .I am pursuing my M.E. in Gujarat Technological University

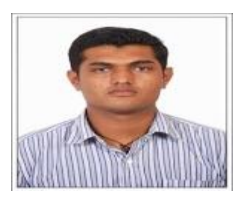

I have completed my B.E. of Electronics \& Communication Engineering in Gujarat Technological University .I am pursuing my M.E. in Gujarat Technological University

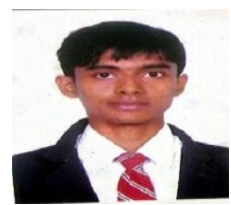

I have completed my B.E. of Electronics \& Communication Engineering in Gujarat Technological University .I am pursuing my M.E. in Gujarat Technological University 